\title{
Imaging Magnetic Nanoparticle Distributions by Atomic Magnetometry-Based Susceptometry
}

\author{
Simone Colombo ${ }^{\circledR}$, Victor Lebedev, Alexey Tonyushkin ${ }^{\circledR}$, Simone Pengue, and Antoine Weis
}

\begin{abstract}
We introduce a Magnetic Particle Imaging Susceptometer (MPIS) that uses a high-sensitivity atomic magnetometer (AM) for recording the spatial distribution of fluid-suspended magnetic nanoparticles. We have evaluated the MPIS performance by one-dimensional scans of structured nanoparticle phantoms, demonstrating, in particular, resolutions of $\approx 2.5 \mathrm{~mm}$ prior to deconvolution and $\ll 1 \mathrm{~mm}$ after deconvolution. Our instrument conceptually follows the general principle of Magnetic Particle Imaging (MPI) for encoding spatial distributions into magnetic flux density variations. Conversely to previously demonstrated MPI methods, MPIS works in time-space by recording time series of the sample's magnetic response including all Fourier components. The device deploys a specifically designed system of coils, a low-frequency excitation scheme, and a simple source localization algorithm. The difference of the AM's frequency response with respect to the conventional receive coil detection allows us to work at much lower driving frequencies. We demonstrate operation at frequencies on the order of $100 \mathrm{~Hz}$, enabling the beneficial use of larger nanoparticles. The spatial distribution encoded into the particles' susceptibility needs a much lower excitation field amplitude compared to conventional MPI scanners. These two features make MPIS least harmful for biological samples and subjects compared to conventional MPI scanners. We also address performance characteristics and other possible applications of MPIS.
\end{abstract}

Index Terms-Biomedical imaging, magnetic particle imaging (MPI), magnetic nanoparticles, atomic magnetometer, very low (VLF), magnetic field, magnetic field compensation, peripheral nerve stimulation (PNS), specific absorption rate (SAR).

This work was supported by the Swiss National Sci-ence Foundation under Grant 200020 162988. (Corresponding author: Simone Colombo.)

S. Colombo is with the Physics Department, University of Fribourg, 1700 Fribourg, Switzerland, and also with the Research Laboratory of Electronics, MIT-Harvard Center for Ultracold Atoms, Department of Physics, Massachusetts Institute of Technology, Cambridge, MA 02139 USA (e-mail: colombos@ mit.edu).

V. Lebedev is with the Physics Department, University of Fribourg, 1700 Fribourg, Switzerland, and also with AG 8.21 Magnetooptische Metrologie, Physikalisch-Technische Bundesanstalt, D-10587 Berlin, Germany.

S. Pengue is with the Physics Department, University of Fribourg, 1700 Fribourg, Switzerland, and also with Biozentrum, University of Basel, CH-4056 Basel, Switzerland.

A. Weis is with the Physics Department, University of Fribourg, 1700 Fribourg, Switzerland.

A. Tonyushkin is with the Physics Department, University of Massachusetts Boston, Boston, MA 02125-3393 USA.

\section{INTRODUCTION}

$\mathbf{H}$ IGH performance atomic magnetometers (AMs) [1] are widely deployed as ambient field detectors/monitors in fundamental physics experiments, such as searches for electric dipole moments of elementary particles [2] or exotic physics [3] as well as in large-scale mapping applications for the surveillance of geomagnetic or outer space fields [4]. Applications to source localization from magnetic field maps on the centimeter-scale were only addressed recently, resulting in spectacular achievements in magnetocardiography [5]-[7], magnetoencephalography [8]-[12], nerve activity detection [13], ultra-low field MRI [14], or the imaging of magnetic nanoparticles [15]-[17].

The latter applications rely on the high sensitivity of AMs in the DC and low-frequency domain and profit from their compact design and room temperature operation. Superconducting interference devices (SQUIDs), while sensitive at low frequencies, have drawbacks of high cost and use of liquid helium. Inductive sensors while cheap, have poor sensitivity at low frequencies. AMs have the potential to be cheap, compact while having low frequency sensitivity rivaling SQUIDs [18], [19]. AMs have only rarely been operated at frequencies [20]-[23] or bandwidths [24] exceeding the low $\mathrm{kHz}$ range, due on one hand to the increasing complexity of high-bandwidth low-noise data acquisition in the multihundred $\mathrm{kHz}$ range and, on the other hand, to the tight competition in that domain with pick-up coil sensors based of Faraday's induction law [25], whose sensitivity increases with the oscillation frequency of the magnetic field of interest.

The potential of replacing pick-up coils by AMs in highfrequency applications was successfully demonstrated for low-field MRI [14] with a sophisticated mode of operation involving magnetic flux guides and flux transformers. AMs, although being extremely competitive in terms of sensitivity with magnetometers using superconducting interference devices (SQUIDs) [18], [19], suffer from a comparatively low tolerance to magnetic field gradients that occur, e.g., in fringe fields of nearby strong field regions. This drawback presents a major obstacle for various emerging applications.

Here we describe an AM system designed to operate as detector in a Magnetic Particle Imaging (MPI) device [26], where it replaces the conventionally used pick-up coil receiver. MPI relies on exciting superparamagnetic iron oxide nanoparticles (SPIONs) by time-varying magnetic fields of tens of $\mathrm{mT}$ in the presence of an inhomogeneous field with a 
gradient of a few $\mathrm{T} / \mathrm{m}$. The challenge consists in recording the response signal of the SPION sample with a sub-nT amplitude in presence of the fringe field from the gradient coil. Colombo et al. [27] have proposed and characterized a coil and AM-sensor design able to generate magnetic field gradients and offsets in the order of $\mathrm{T} / \mathrm{m}$ and $\mathrm{mT}$, respectively, without significantly affecting the AM's performances. Using that approach we demonstrate an AM-based signal detection which can compete with, and in some respects outperform, the conventional MPI detection by pick-up coils. The presented device demonstrates a combination of high robustness against magnetic field perturbations and field gradients as well as a large bandwidth, while maintaining a high magnetometric sensitivity.

The spatial resolution of an MPI scanner uses the fact that an oscillatory response signal is only produced by SPIONs located in a region around the zero-field point (or line) in the inhomogeneous field. Scanning is achieved by moving the field-free point, FFP (or field-free line, FFL [28]), through the sample volume, thus allowing one to map the SPIONs' spatial distribution with $\mathrm{mm}$ resolution.

Since SPIONs have a high biocompatibility and are relatively easy to produce, they have already been deployed in a number of biomedical applications [29], [30]. SPIONs can be functionalized in view of their selective interaction with biological entities (cells, organs, tissue, tumors) aiming at targeted drug delivery. Their surface can be modified to maintain stability in chemically aggressive biological environments, and their susceptibility and Curie temperature can be readily tuned for hyperthermia applications. For such applications MPI provides a key imaging/monitoring tool for nanomedicine, being in that sense a diagnostic and therapeutic technique.

Recently, various research groups have reported dynamical imaging of cardiovascular systems in small animals by MPI, which, in combination with conventional MRI, delivers structural data with functional information [31], [32], thereby yielding a safer alternative [33] to positron-emission tomography assisted MRI [34]. Such small-animal sized scanners have become commercially available [35], [36]. In contrast to experiments on small animals, MPI scanners for humans face the serious challenge of compliance with patient safety regulations in connection with the exposure to high-power, high-frequency electromagnetic fields [33], [37]-[39].

MPI scanners deployed so far rely on the encoding of magnetic moment distributions into the amplitudes of harmonics (overtones) in the SPIONs' response signal induced by a strong monochromatic drive (excitation) field. The encoded information has to be extracted from the detected (discrete harmonics) signals by demanding calibration and computation algorithms that currently prevent a desirable live source reconstruction. We note that some specific modalities [40] allow for such a live readout.

Our use of a specifically adapted AM changes the above principle of operation of MPI scanning and yields a multimodal instrument which we refer to as magnetic particle imaging susceptometer, MPIS. The MPIS delivers unfiltered time domain data (containing full spectral information) of the magnetic flux density (response) produced by the SPION

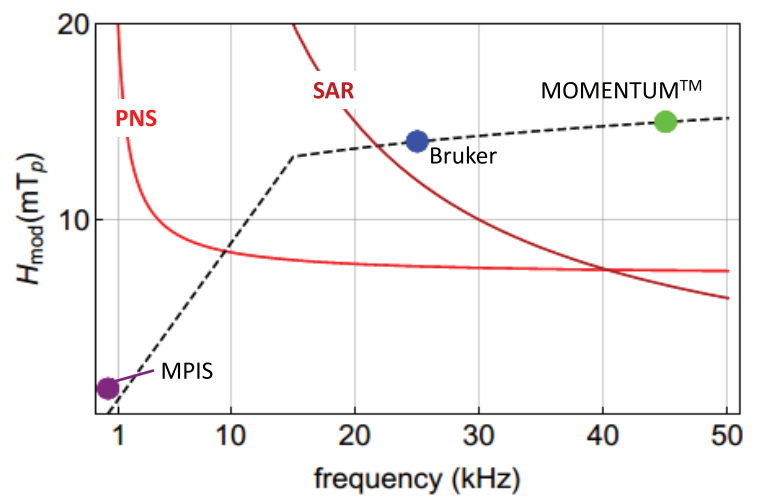

Fig. 1. Graphical representation of PNS and SAR limits [33] as a function of modulation field peak amplitude and frequency. The purple dot denotes the operation condition of the MPIS system presented in this paper, while the blue and green dots the operation conditions of the two available preclinical MPI scanners Bruker [35] and MOMENTUM ${ }^{\mathrm{TM}}$ [36], [42], respectively. The dashed line represents the scanner operation with a modulation amplitude of $H_{\text {mod }}=10 H_{k}^{\mathrm{opt}}$, where $H_{k}^{\mathrm{opt}}$ is the characteristic field of 'optimal' SPION size, assuming a Langevin-Brown particle, for the corresponding frequency; The kink at $\approx 14 \mathrm{kHz}$ denotes the transition from a dominant Néel relaxation (low frequencies) regime to a Brown (larger frequencies) relaxation dominated one.

sample at low frequencies, allowing a continuous tomographic tracking of the sample magnetization. The capability of lowfrequency operation in particular is a main advantage regarding safety restrictions. Moreover, the direct magnetic moment distribution scanning significantly simplifies source reconstruction, and the direct detection of the magnetic susceptibility, rather than its anharmonic components, relaxes the demand on the strength of the SPION-saturating fields and steepness of the gradient.

As a result, we present a proof-of-principle AM-based MPIS, built with a simple magnetic coil system and a compact AM module. Our imaging instrument is able to compete with state-of-the-art MPI scanners in terms of resolution, field of view and sensitivity. Moreover, as shown in Fig. 1, it is free from some of their most severe constraints, such as particle size limitations, Specific Absorption Rate (SAR) limits and Peripheral Nerve Stimulation (PNS) effects [41].

In this paper we address a one-dimensional mechanical scanner as the simplest implementation of an MPIS instrument, focusing on its design and performance. To facilitate comparison with conventional MPI modalities we have chosen the Resovist ${ }^{\circledR}$ tracer as test SPION material.

The paper is organized as follows: Sec. II describes in detail conventional MPI operation and its modifications for AMbased MPIS; Sec. III reviews the magnetic system design of the MPIS; in Sec. IV the design and mode of operation of the AM are discussed, Sec. V presents the detected magnetic field patterns and reconstructed SPION distributions, and Sec. VI discusses in detail the MPIS performance in terms of detection limit and operational bandwidth.

\section{Magnetic Nanoparticle IMAGing}

\section{Susceptometry With AN Atomic Magnetometer}

The detection of SPIONs by MPI is based on the nonlinear response of their magnetization $M$ to an external magnetic 
field $H$. The magnetization curve $M(H)$ of an ensemble of magnetic nanoparticles has a superparamagnetic character, characterized by a (quasistatic) saturation field of a few $\mathrm{mT} / \mu_{0}$ [43] and a vanishing coercitivity (no hysteresis in quasistatic fields). MPI is essentially a dynamic technique that is applied to fluid SPION suspensions in a frequency range where viscous drag does not significantly affect the particles' response to the driving field. In our experiments we limit ourself to the DC to low-frequency domain of applied magnetic fields, where the SPIONs' response follows a Langevin-Brown model [44]. However, this model has to be applied with caution for faster drives by stronger fields [45].

In a first order approximation the characteristic field is given by $H_{k}=k_{\mathrm{B}} T /\left(\begin{array}{ll}\mu_{0} & V_{\text {core }} M_{s}\end{array}\right)$, where $V_{\text {core }}$ is the particles' core volume, $M_{S}$ the core saturation magnetization and $T$ the sample temperature. When the sample is exposed to a modulation field $\vec{H}_{\text {mod }}(t)$ that harmonically oscillates with a small amplitude $H_{\bmod } \ll H_{k}$, it will show a positive (paramagnetic) AC-susceptibility

$$
\chi_{\mathrm{AC}} \propto \frac{\mathrm{d} \vec{M}\left(\vec{H}_{\mathrm{off}}\right)}{\mathrm{d} H} \cdot \hat{H}_{\mathrm{mod}} \quad \text { with } \hat{H}_{\mathrm{mod}} \equiv \frac{\vec{H}_{\mathrm{mod}}}{\left|\vec{H}_{\mathrm{mod}}\right|}
$$

so that $\chi_{\mathrm{AC}} \rightarrow 0$ in large offset fields $\left|H_{\mathrm{off}}\right| \gg H_{k}$.

From the above it follows that in a spatially distributed sample exposed to a magnetic field gradient, only SPIONs located in a region - referred to as 'blurring kernel' - in which the local field obeys $|H| \ll H_{k}$ contribute to the sample's total AC-susceptibility $\chi_{\mathrm{AC}}$. The recorded AC-susceptibility therefore depends on the location $\vec{r}_{s}$ of the FFP (FFL) in the sample frame. By scanning $\vec{r}_{s}$ through the sample one can thus infer the spatial SPION distribution. Recording the $\chi_{\mathrm{AC}}\left(\vec{r}_{s}\right)$ dependence results in a blurred image of the SPIONs' spatial distribution. The extension and shape of the blurring kernel are defined by the sample's characteristic field $H_{k}$ and the gradient strength together with the DC magnetic field pattern and the orientation of the AC modulation field.

At sufficiently low frequencies (for which the SPIONs' magnetization follows the excitation field without time lag), the AC-susceptibility $\chi_{\mathrm{AC}}=\mathrm{d} M_{\mathrm{tot}} / \mathrm{d} H$ of a homogeneous SPION distribution with density $n_{\text {tot }}$ exposed to a static offset field $H_{\mathrm{off}}$ is given by

$$
\chi_{\mathrm{AC}}\left(H_{\mathrm{off}}\right)=n_{\mathrm{tot}} \frac{M_{s} V_{\text {core }}}{H_{k}} L^{\prime}\left(h=\frac{H_{\mathrm{off}}}{H_{k}}\right),
$$

where $L^{\prime}(h) \equiv \mathrm{d} L / \mathrm{d} h=h^{-2}-\operatorname{csch}^{2}(h)$ is the derivative of the Langevin function $L(h)$. The last equation is valid also in the case of an inhomogeneous SPION distribution, where the density $n_{\text {tot }}$ is given by the total mass divided by the total volume.

In the presence of a magnetic field gradient $G_{x}=d H / d x$ in the $x$-direction one has a selection field

$$
H_{\mathrm{off}}(x)=H_{\mathrm{off}}^{\mathrm{DC}}+H_{\mathrm{G}}=G_{x}\left(x-x_{s}\right),
$$

so that SPIONs with a (normalized) spatial distribution $s(x)$ will contribute to the total AC-susceptibility as

$$
\chi_{\mathrm{AC}}=\int \delta \chi_{\mathrm{AC}}(x)=\int_{-\infty}^{\infty} s(x) \chi_{\mathrm{AC}}\left(H_{\mathrm{off}}(x)\right) \mathrm{d} x .
$$
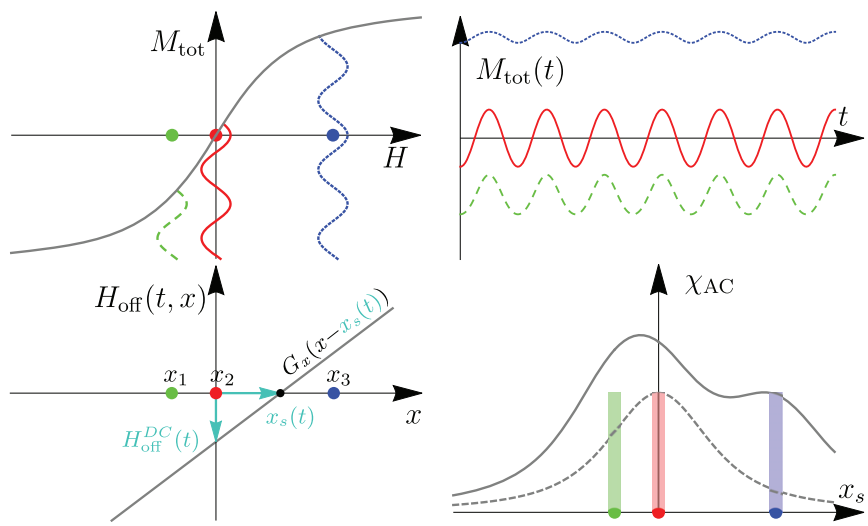

Fig. 2. Basics of 1D-MPIS. Top: $M_{\text {tot }}(H)$ magnetization curve (left) with time-dependent magnetization (right) induced by harmonic excitation superposed on offset fields $H_{\text {off }}^{\mathrm{DC}}=0$ (red solid line), $H_{\text {off }}>0$ (blue dotted line), and $H_{\text {off }}<0$ (green dashed line). The top right graph shows the oscillatory responses of the magnetization in the three cases. Bottom, left: A linear field gradient superimposed on the offset field $H_{\text {off }}^{\mathrm{DC}}$ generates a field free point (FFP), whose location $x_{S}$ is scanned by scanning $H_{\text {off }}^{\mathrm{DC}}$. Demodulation of $M_{\text {tot }}$ at the oscillation frequency of the modulation field $H_{\text {mod }}$ yields the total AC-susceptibility function $\chi_{\mathrm{AC}}\left(x_{S}\right)$ (bottom, right). This function is the convolution of the SPION distribution $s(x)$ (colored bars) with the blurring kernel (dashed curve), which represents the weights with which particles at different locations (here three pointlike SPIONs at positions $x_{1 \ldots 3}$, marked by colored dots) contribute.

In $3 x_{s}$ denotes the location of the FFP. The latter allows us to express the AC-susceptibility as a function of the FFP position $x_{s}$ by the convolution

$$
\chi_{\mathrm{AC}}\left(x_{s}\right) \approx n_{\mathrm{tot}} \frac{M_{s} V_{\text {core }}}{H_{k}} \int_{\mathrm{FOV}} L^{\prime}\left(\frac{G_{x}}{H_{k}}\left(x_{s}-x\right)\right) s(x) \mathrm{d} x
$$

of the density distribution $s(x)$ with the weighting function (2) which represents the blurring kernel introduced above.

The FFP is scanned through the SPION sample by ramping the amplitude of a homogeneous field offset field $H_{\text {off }}^{\mathrm{DC}}$ that is superimposed onto the gradient field $H_{G}$. Figure 2 illustrates the principle of this magnetic scan in one dimension. Alternatively one may perform the scan by setting $H_{\mathrm{off}}^{\mathrm{DC}} \equiv 0$, and moving the sample by mechanical means through the FFL (mechanical scan). When seen from the sample's reference frame the situation in that case is analogous to the magnetic scan presented in Fig. 2.

As depicted in the bottom of Fig. 2, the resulting $\chi_{\mathrm{AC}}\left(x_{s}\right)$ is a blurred image of the SPION density distribution $s(x)$. Without source reconstruction (by deconvolution or other methods), the spatial resolution is thus determined by the width $\delta x=4.16 H_{k} / G_{x}$ of the blurring kernel, which depends both on the gradient strength $G_{x}$ and the SPION characteristic field $H_{\mathrm{k}}$.

The SPIONs' oscillating magnetization is detected by an atomic magnetometer (AM) located in close vicinity of the sample (see Fig. 3). The signal of interest is the amplitude $\delta B_{\chi_{A C}} \propto M_{\text {tot }}(t)$ of the magnetic flux density component at the sensor location which oscillates at the excitation frequency $f_{\text {mod, viz., }}$

$$
\delta B_{\chi_{\mathrm{AC}}}\left(H_{\mathrm{mod}}, x_{s}\right)=\frac{\mu_{0}}{4 \pi} \frac{\chi_{\mathrm{AC}}\left(x_{s}\right) H_{\mathrm{mod}}}{z_{\mathrm{AM}}^{3}},
$$


where $z_{\mathrm{AM}}$ the distance between the AM and the SPION sample, assuming a sample with a size $\ll z \mathrm{AM}$.

The signal delivered by the used atomic magnetometer is proportional to $B_{\mathrm{AM}}=\left|\vec{B}_{\mathrm{AM}}\right|$, where the components of $\vec{B}_{\mathrm{AM}}$ are averages over the AM's sensitive volume. The AM's mode of operation requires the application of a small $(\ll \mathrm{mT})$ static holding field $\vec{B}_{0}=B_{0} \hat{e}_{i}$ (oriented along the spatial direction $\hat{e}_{i}$ ) that is much larger than the field of interest $\delta \vec{B}_{\chi_{\mathrm{AC}}}$. The latter (vectorially) adds to $\vec{B}_{0}$, so that

$$
B_{\mathrm{AM}}=\left|\vec{B}_{0}+\delta \vec{B}_{\chi_{\mathrm{AC}}}\right| \approx B_{0}+\delta B_{\|}+\mathcal{O}\left(\left|\delta \vec{B}_{\chi_{\mathrm{AC}}}\right|^{2} / B_{0}\right),
$$

where $\delta B_{\|}=\delta \vec{B}_{\chi_{\mathrm{AC}}} \cdot \hat{B}_{0}=\delta \vec{B}_{\chi_{\mathrm{AC}}} \cdot \hat{e}_{i}$ is the projection of the field of interest $\delta \vec{B}_{\chi_{\mathrm{AC}}}$ onto the holding field (Sec. IV). In order to maximize the signal $\propto \delta B_{\|}$one therefore has to orient the holding field $\vec{B}_{0}$ along the anticipated direction of the flux density $\delta \vec{B}_{\chi_{\mathrm{AC}}}$ at the AM location.

In our scanner the AM is mounted outside of the SPION magnetizing coil system, so that it feels the fringe fields of the latter. These fields and their gradients have to be carefully compensated by auxilliary coils in order to properly define the magnitude and orientation of the AM holding field needed for optimal SPION detection (Sec. III). The compensation fields have a negligible effect on the total field experienced by the SPION sample.

\section{A. Comparison to Frequency-Space and x-Space MPI}

As described above, MPIS detects the temporal change of the susceptibility $\chi_{\mathrm{AC}}(t)$ that results from the temporal change of the gradient configuration, characterized by the FFL position $x(t)$. Having a calibrated measure of $x(t)$ yields a representation of $\chi_{\mathrm{AC}}(x)$, which is the blurred image of the sample's density distribution $s(x)$. By simple deconvolutionsimilarly to the one deployed in X-space MPI [40]-MPIS then yields the (unblurred) density distribution $s(x)$. In contrast to conventional MPI [26], [46], [47], MPIS records the sample's instantaneous AC susceptibility in a way that is independent of the trajectory and dynamics of the FFP motion. Moreover, source reconstruction with MPIS is more efficient and more robust than the conventionally used (and sometimes ill-posed) inverse problem solution methods. Recording the total magnetization provides data on the linear and non-linear parts of the AC susceptibility, the former one not being accessible to the conventional pick-up coil detection, in which the linear part is encoded in the (filtered-out) fundamental of the MPI signal.

The amplitude of an AM-detected signal is frequencyindependent (down to DC) in contrast to receive coil signals, whose amplitude drops linearly with decreasing frequency. Operation at lower frequencies allows the use of larger-sized SPIONs, yielding larger signals as well as a higher spatial resolution (narrower blurring kernel), thus offering a clear advantage over the conventional receive coil detection [48]-[51].

With our method, decoupling the FFP scan from the drive field oscillation delivers a $\chi_{A C}$-signal which does not depend on the speed at which the FFP is moved, thus circumventing the velocity compensation problem of X-space MPI [40]. The decoupling is most efficient with magnetic sensors that can detect DC, or low-frequency fields, such as fluxgates, SQUIDs, or, as in our case, AMs. The choice of a broadband sensor also results in a greater experimental flexibility, since the drive field frequency $f_{\text {mod }}$ and the scan speed can be easily and independently adapted to experimental conditions.

AM detection has a finite upper frequency which constrains the maximal achievable scan speed as will be addressed in Sec. VI-C. It can, however, be combined with any of the classical (X-space or frequency-space) modes of scanner operation to boost the imaging speed by coupling the FFP motion and AC modulation, and by analyzing the AM signal in the same way as in conventional MPI. Below, we therefore address in detail the direct scan mode of the SPION's AC susceptibility, in order to provide deeper and clearer insights into the operation of the AM sensing.

\section{COIL SYSTEM}

The coil system, shown in Fig. 3, is composed of the main (selection) coil, which defines the nanoparticles' magnetization, the modulation coil, and compensation coils needed to suppress the stray fields and gradients from the former coils at the AM location. The selection and modulation fields are produced by means of elongated racetrack coils [52], [53] whose field - at the sample location - is well described as the field produced by two sets of four infinitely long wires. The straight wire approximation yields an analytical expression for the magnetic field produced by each coil pair, thus allowing us to model the sample response and, in consequence, to rapidly reconstruct the particle distribution from the detected signal. In this design, the field variations along the y-axis are negligible (on the scale of the sample extension) and the selection coil thus produces a highly homogeneous FFL extending along that axis. As described below we have (mechanically) scanned SPION samples (elongated along the y-axis) by moving them along the $\mathrm{x}$-axis.

The compensation coils were designed using the following procedure: We calculate the flux density produced by the SPION selection/modulation coils — modeled as straight wires - at the AM sensor position. For compensating the fringe field from the modulation coil we use an auxiliary pair of infinitely long wires that are separated by $\Delta x_{\text {mod }}$ and are located at a distance $z_{\bmod }$ below the FFL in the center of the main racetrack coils. Both wires carry identical currents $\alpha_{\text {mod }} I_{\text {mod }}$ flowing in the same direction, $I_{\text {mod }}$ being the current in the modulation coil. We use the same two-wire approach for designing the compensation of the selection coil's fringe field. Here, the two wires are separated by $\Delta x_{\text {sel }}$ and located at a distance $z_{\text {sel }}$ below the FFL. These wires carry oppositely-flowing currents $\alpha_{\text {sel }} I_{\text {sel }}, I_{\text {sel }}$ being the selection coil current. In their experimental implementation, both twowire compensation coils are laid out as elongated race track coils as shown in Fig. 3.

Using a least squares fit method, we then fit the parameters $\Delta x_{\text {mod }}, \Delta x_{\text {sel }}, \alpha_{\text {mod }}$, and $\alpha_{\text {sel }}$, such that the compensation coils completely cancel the field and its first-order gradient at the AM sensor location. For the fit we have imposed that $\alpha_{\text {mod }}$ and $\alpha_{\text {sel }}$ are integer numbers that represent in practice the 


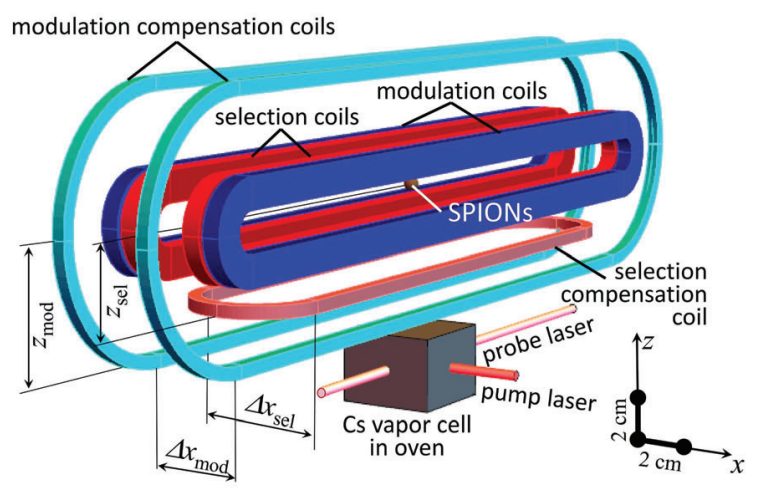

Fig. 3. Three dimensional sketch of the coil-magnetometer system used for the experiments described in this section. The key coil system dimensions are $\Delta x_{\text {sel }}=43 \mathrm{~mm}, \Delta x_{\text {mod }}=27 \mathrm{~mm}, z_{\text {sel }}=39 \mathrm{~mm}$, and $z_{\mathrm{mod}}=46 \mathrm{~mm}$.

number of loops in the corresponding coils. In this way we obtain a self-compensating coil system that is independent of the currents in the SPION selection and modulation coils. Moreover, the system also strongly suppresses effects from current noise, when each compensation coil is wired in series with the corresponding main coil.

As a result, only two power amplifiers are needed to drive all SPION manipulating coils. The spectral noise density of $B^{\text {tot }}$ measured under typical experimental conditions at the AM sensor location is

$$
\delta B_{\text {noise }}^{\text {tot }}<2 \frac{\mathrm{pT}_{\mathrm{rms}}}{\sqrt{\mathrm{Hz}}}
$$

which is comparable to (or even smaller than) the sensitivity $\delta B_{\mathrm{NEM}}$ of the atomic magnetometer.

Since the main coils heat up appreciably during operation, the ensuing thermal expansions degrade the quality of the modulation field compensation. We have therefore implemented a fine-compensation of the leaked field $\delta B_{\text {mod }}$ by winding one extra independent wire loop on the modulation compensation coil whose current is adjusted by hand using a voltage-controlled current source. The extra noise introduced by this fine-compensation is negligible compared to the AM sensitivity. We could not observe any obvious effects from acoustical or mechanical instabilities on the performance of the magnetometer, apart from ones that result from the setup's thermal expansion. The whole setup was mounted on an aluminum board damped by Sorbothane sheets, and placed on a massive wooden support that efficiently suppresses mechanical perturbations of the system.

For all measurements reported below we have powered the modulation coil system by an AE Techron 7224 DCAC amplifier operated in constant-current mode, while the selection coil system was driven by an EA-PSI 9080-100 lownoise DC power supply. The fine compensation was powered by a Thorlabs LDC205C voltage-controlled current source.

\section{Atomic Magnetometer}

The AM's working principle is based on the Larmor precession - induced by an external field $B_{0}-$ of the magnetization produced by a spin-polarized vapor of (paramagnetic) ${ }^{133} \mathrm{Cs}$ atoms.

In a quantum picture, the spin polarization, i.e., the macroscopic magnetization of the vapor corresponds to an imbalance of the atomic ground states' magnetic sublevel populations. This imbalance is produced by optical pumping [54] with circularly-polarized resonance light, a method that consists in transferring angular momentum quanta from the photons to the atoms by incoherent light scattering. We refer to the light beam used to produce the magnetization as pump beam. In our geometry it propagates along the direction of the local holding field $B_{0}$.

In external magnetic fields, the resulting macroscopic magnetization behaves in a classical manner: When the magnetization is not parallel to the magnetic field, it will precesses around the latter at the Larmor frequency $f_{L}=\gamma_{F}\left|\vec{B}_{0}\right|$, where $\gamma_{F} \approx 3.5 \mathrm{~Hz} / \mathrm{nT}$ for ${ }^{133} \mathrm{Cs}$. The polarized vapor is exposed to a weak radio-frequency magnetic field that is perpendicular to $\vec{B}_{0}$ and oscillates at a frequency $f_{R F} \approx f_{L}$. In this conventional magnetic resonance geometry, the RF field coherently drives the atomic magnetization around the magnetic field.

In the AM, also called optical magnetometer, the precessing magnetization is read out by optical means. For this one uses the fact that the (resonant) optical properties (complex dielectric susceptibility) of the atomic vapor depends on the degree and orientation of its spin polarization (magnetization). The axial vector nature of the magnetization breaks the isotropy of the vapor, which exhibits both circular dichroism (CD) and circular birefringence (CB) in consequence. The precessing magnetization can be monitored by an appropriately polarized and tuned weak probe beam that propagates at right angles with respect to the pump beam and which measures either the $\mathrm{CD}$ or the $\mathrm{CB}$.

The phase between the driving RF-field and the corresponding oscillation of the transmitted probe power depends on the detuning between $f_{L}$ and $f_{R F}$. When $f_{L} \approx f_{R F}$, any field change $\delta \overrightarrow{\mathrm{B}}$ will thus induce a phase change $\delta \varphi \propto f_{L}-f_{R F}$. As described below, it is possible to implement a negative feedback loop that keeps the phase at a constant preset value by adjusting $f_{R F}$ in accordance with the change of $f_{L}$. In this way the RF frequency can be locked to the Larmor frequency $f_{L} \propto\left|\vec{B}_{0}+\delta \overrightarrow{\mathrm{B}}\right|$. We note that the magnetic flux offset $B_{0}$ needed for the AM operation is on the order of $10 \mu \mathrm{T}$, i.e., $100-1000$ times smaller than field used to manipulate the SPIONs' magnetization.

Details of the magnetometer deployed in this work are described in [27]. It is a pump-probe $M_{x}$-magnetometer operated in the phase-locked-loop mode. The cesium vapor is produced by a heated droplet of metallic cesium contained in a small sidearm of a cubic glass cell together with $\approx 20$ mbar of argon buffer gas. The intersecting pump and probe beams define a relatively small sensing volume of $\approx 1 \mathrm{~mm}^{3}$ (up to a small diffusion length correction) which reduces the device's sensitivity to magnetic field gradients that originate from the SPION excitation coils and the overall ambient field. The cell is mounted in a small oven heated to an optimized temperature of $55^{\circ} \mathrm{C}$. 


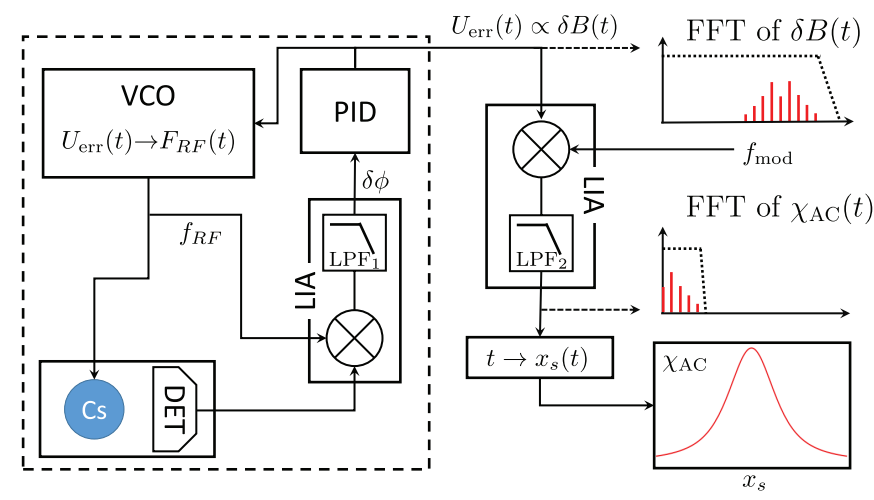

Fig. 4. MPIS detection scheme. The dashed box shows the PLL operation of the AM. LIA: lockin amplifier; $\operatorname{LPF}_{1,2}$ : Low-pass filters; $\mathrm{DET}$ : detection of spin precession. PID: proportional-integral-derivative controller. VCO: voltage-controlled oscillator.

We empirically find a maximal sensor sensitivity when

- the circularly-polarized pump beam is resonant with the $F=3 \rightarrow F=4$ hyperfine component of the Cs $D_{2}$ transition, and

- the spin polarization is inferred by detecting the paramagnetic rotation angle of the linearly-polarized probe beam, whose frequency is blue-detuned by $\approx 1.3 \mathrm{GHz}$ with respect to the $F=4 \rightarrow F=5$ hyperfine component of the Cs $D_{1}$ transition.

We have further optimized the power of the pump and probe beams as well as the cesium vapor pressure (cell temperature), which are all interdependent. We used a pump power of $\approx 6 \mathrm{~mW}$, and a vapor pressure such that $\approx 50 \%$ of the probe beam power is absorbed by the cell.

We note that the pump beam depletes the populations of the $F=3$ hyperfine ground state level, so that most of atoms are in the $F=4$ state, the only hyperfine state which contributes to the signal detected by the probe beam. Since the hyperfine splitting of the excited state of the $D_{2}$ transition is spectrally not resolved, we tune the laser to its blue side - near the closed $F=4 \rightarrow F=5$ transition - such that there is a $90 \%$ probability (estimated by a simulation calculation) that the excited $6 \mathrm{P}_{3 / 2}$ state relaxes back to the $F=4$ hyperfine level of the $6 S_{1 / 2}$ ground state.

As already mentioned, we operated the magnetometer in a feedback mode, whose experimental realization is outlined in Fig. 4. The $\delta B(t)$ signal is inferred from the temporal variations $\delta f(t)$ of the RF-frequency $f_{R F}(t)$, that is phaselocked to the Larmor frequency $f_{\mathrm{L}}(t)$. Since $\delta f \ll f_{R F} \approx f_{\mathrm{L}}$, we subtract the Larmor frequency $f_{\mathrm{L}}^{(0)}$, measured with switched-off SPION excitation and modulation coils, from the actual RF-frequency, i.e., $\delta f(t)=f_{R F}(t)-f_{\mathrm{L}}^{(0)}$. These frequency variations are then converted into a voltage $\delta V(t)=$ $\alpha_{F V C} \delta f(t)$ by means of a linear frequency-to-voltage converter with an adjustable conversion factor $\alpha_{F V C}$. Finally, we obtain the magnetic flux density change from $\delta B(t)=$ $\delta V /\left(\gamma_{F} \alpha_{F} V C\right)$.

Because of technical reasons, the above feedback loop operates only in a certain band of frequencies $f_{R F}$ that we have (arbitrarily) limited for the experiments described below to the range $f_{\mathrm{L}}^{(0)} \pm 10 \mathrm{kHz}$ in order to ensure an optimal performance of the phase detector. The latter range corresponds to a range $\delta B \approx \pm 3 \mu \mathrm{T}$ of detectable field deviations. This type of constraint may be altered by suitable choice of the phase detector parameters and the value of the holding field, i.e., of the corresponding $f_{\mathrm{L}}^{(0)}$. A more fundamental limit is imposed by the fact, that an AM of the presented type cannot be operated in a field close to or larger than the Earth field due to the quadratic Zeeman shift of the magnetic sublevels. This feature limits the actually achievable detection range to a few ten $\mu \mathrm{T}$, leading to a performance degradation or malfunction otherwise.

\section{Results}

\section{A. Point Spread Function of 1D Mechanical AM-MPIS}

In order to demonstrate the AM-MPIS technique we have recorded a one-dimensional scan of a SPION sample by moving the sample along the $x$-axis (Fig. 3). The sample is mounted on a homebuilt rail-guided carriage that allows moving it through a FOV of $\pm 15 \mathrm{~mm}$, the FFL/FFP being located ax $x=0$. A simple position encoder with a transducer converting the translation into a rotation driving a potentiometer is used to monitor the position $x$ in terms of a voltage.

The Point Spread Function (PSF) of this 1D mechanical scanner, which represents the blurring kernel introduced above can be calculated from the field pattern produced by the selection and modulation coils at the sample position. As described by Colombo et al. [27], the field from the finite-sized coils was calculated numerically by boundary element methods using the Radia [55] software package for Mathematica ${ }^{\circledR}$ (Wolfram).

The PSF of the 1D mechanical scanner variant is given by the change $\delta B_{\chi_{\mathrm{AC}}}$ of the flux density component along the AM holding field $\vec{B}_{0}$ that is produced by a point-like sample located at the position $x=0$. The magnetic dipole formula and Eq. (5) with $s(x)=\delta(x)$ yield

$$
\begin{aligned}
\operatorname{PSF}_{\text {mech }}(x) \equiv & \delta B_{\chi_{\mathrm{AC}}} \\
= & n_{\text {tot }} \mu_{\text {core }} \frac{\mu_{0}}{4 \pi} \frac{z_{\mathrm{AM}}^{2}-2 x^{2}}{\left(z_{\mathrm{AM}}^{2}+x^{2}\right)^{5 / 2}} \\
& \times \frac{\mathrm{d} H_{\text {mod }}(x)}{\mathrm{d} I_{\text {mod }}} \frac{I_{\text {mod }}}{H_{k}} L^{\prime}\left(\frac{\mathrm{d} H_{\text {sel }}(x)}{\mathrm{d} I_{\text {sel }}} \frac{I_{\text {sel }}}{H_{k}}\right),
\end{aligned}
$$

We have used the fact that the atomic magnetometer is located at the coordinate $\left(0,0, z^{\mathrm{AM}}\right)$ with respect to the center of the main coils, and that it is sensitive only to the flux density component parallel to the $x$-axis.

We note that in the case of a mechanical scanner, the PSF needs to be unambiguously defined over an interval which exceeds the FOV by an amount that depends on the sample extension and the effective width of the PSF. In the system presented here the FOV covers a range of $24 \mathrm{~mm}$, resulting in the need of a PSF defined for $x \in(-24 \mathrm{~mm},+24 \mathrm{~mm})$.

\section{B. Scan of the Point-Like Sample}

Figure 5 shows the result of a 1D-scan of a $3.5 \mathrm{~mm}$ inner diameter capillary filled with $\approx 140 \mu$ l of undiluted Resovist $^{\circledR}$ containing a total of $\approx 3.9 \mathrm{mg}_{\mathrm{Fe}}$. The $15 \mathrm{~mm}$ long 


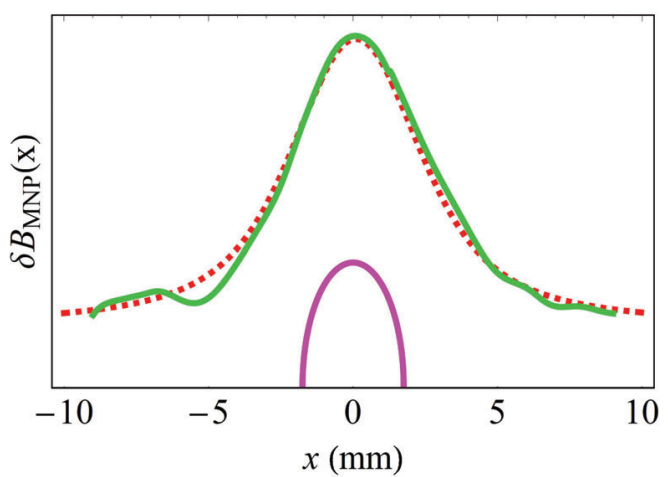

Fig. 5. The solid green curve shows the flux density change recorded by the AM during a one dimensional mechanical scan of a cylindrical sample sample of $140 \mu \mathrm{l}$ of Resovist ${ }^{\circledR}$ with radius $r_{i}=1.75 \mathrm{~mm}$. The dashed red line is the expected signal obtained by convolving the sample's spatial distribution with the PSF given by (9). The magenta line denotes the SPIONs' spatial distribution $s(x)$ described in (10).

capillary is oriented along the FFL, and moved along the $x$-axis, transverse to the FFL. The selection coil system was powered by a DC current $I_{\text {sel }}$ of $10 \mathrm{~A}$, producing a gradient of $\approx 1 \mathrm{~T} / \mathrm{m}$. The modulation system is driven by a current oscillating at $f_{\mathrm{m}}=65 \mathrm{~Hz}$ with a peak amplitude $I_{\bmod }=2 \mathrm{~A}$, producing an oscillating field at $x=0$ with an amplitude of $\approx 1.4 \mathrm{kA} / \mathrm{m}$. The sample was scanned by hand through a FOV of $\approx 20 \mathrm{~mm}$ at an average speed of $10 \mathrm{~mm} / \mathrm{s}$. We simultaneously recorded the sample position $x_{\mathrm{pos}}(t)$ from the encoder and $\delta B(t)$ delivered by the AM during two forwardbackward scans through the FOV.

The recorded data were then analyzed off-line. In particular, we extracted the signal of interest induced by the sample's ACsusceptibility $\delta B_{\chi_{\mathrm{AC}}}(t)$ by demodulating the measured magnetic flux density variation $\delta B(t)$ at the modulation frequency $f_{\mathrm{m}}=65 \mathrm{~Hz}$ using a sinc-filter with a bandwidth of $10 \mathrm{~Hz}$. The FFL position $x_{s}(t)$ in the sample's frame of reference is related to the measured sample position in the laboratory frame $X(t)$ by $x_{s}(t)=-x_{\text {pos }}(t)$. From the time series $\delta B_{\chi_{\mathrm{AC}}}(t)$ and $x_{s}(t)$ it is then straightforward to get a representation of $\delta B_{\chi_{\mathrm{AC}}}\left(x_{s}\right)$. We averaged the four FOV scans in order to get the blurred (convoluted) 1D image of our sample, shown in Fig. 5.

The red dashed line in (Fig:5) is the theoretically expected signal $\delta B_{\text {theo }}\left(x_{s}\right)$ resulting from the convolution of the $\mathrm{PSF}_{\text {mech }}$, given by (9), with the spatial distribution

$$
s(x) \propto \begin{cases}\sqrt{1-\left(x / r_{i}\right)^{2}} & |x| \leq r_{i}=1.75 \mathrm{~mm} \\ 0 & |x|>r_{i},\end{cases}
$$

of the sample along the $\mathrm{x}$-axis, assuming the shape of a disk representing the cross-section through a cylinder (capillary) with inner radius $r_{i}$.

Since our experiments are performed under slowly varying weak magnetizing fields (up to $\approx 10 \mathrm{kA} / \mathrm{m}$ ), the LangevinBrown formalism allows describing the SPION ensemble by a single parameter, $H_{k}^{\text {Res }}$, which for Resovist ${ }^{\circledR}$ is expected to be in the range of few $\mathrm{kA} / \mathrm{m}$ [56].

We have measured this parameter for our samples independently with our AM-based MPS system [57] and cross-checked the results with those of a commercial MPS unit from Pure Devices, and found $H_{k}^{\text {Res }} \approx 1.03 \mathrm{kA} / \mathrm{m}$.
The agreement of the modeled signal with the measured data is remarkable, considering that only two ('hand-adjusted') free parameters were used, viz., $n_{\text {tot }}$, which tunes the signal amplitude, and a flat background that takes the imperfectly compensated fringe modulation field in account. The nice agreement validates not only the signal modeling, but also the MPIS scheme based on atomic magnetometry that we had proposed earlier in Refs. [27] and [58].

\section{Scan of a Structured SPION Sample and Gradient Dependence}

In order to test the spatial resolution of this first MPIS implementation and its dependence on the gradient strength we have produced a sample consisting of three $1.5 \mathrm{~mm}$ inner diameter capillaries, separated (pairwise) by $\approx 6 \mathrm{~mm}$ and each filled with $\approx 50 \mu$ of Resovist $^{\circledR}$, corresponding to an amount of $\approx 1.4 \mathrm{mg}_{\mathrm{Fe}}$ per capillary. The $30 \mathrm{~mm}$ long capillaries lie in one plane and are oriented along the FFL (y-axis) and the whole sample is moved along the $x$-axis. We used a modulation field with an amplitude of $\approx 1.1 \mathrm{kA} / \mathrm{m}$ (at $\mathrm{x}=0$ ) oscillating at $f_{\mathrm{m}}=121 \mathrm{~Hz}$. We performed three measurements similar to the one described above for the single capillary with gradients (at $x=0$ ) of $0.75,1.0$, and $1.4 \mathrm{~T} / \mathrm{m}$, respectively. Each measurement consisted of 16 scans ( 8 forward-backward cycles) performed by manually moving the sample through a FOV of $\approx 22 \mathrm{~mm}$ with an average speed $v_{s}$ of $7.5 \mathrm{~mm} / \mathrm{s}=\mathrm{FOV} / 3 \mathrm{~s}^{-1}$. The demodulation bandwidths (as determined by $\mathrm{LPF}_{2}$ in Fig. 4) were set to $f_{\mathrm{BW}}=5 \mathrm{~Hz}$ for the 0.75 and $1.0 \mathrm{~T} / \mathrm{m}$ gradients and to $8 \mathrm{~Hz}$ for the $1.4 \mathrm{~T} / \mathrm{m}$ gradient.

The three 1D images, each one obtained after averaging the 16 FOV scans, are shown in Fig. 6. We recall that these images represent convolutions of the actual sample geometry with the PSF. We also point out that constant offsets on the order of $\approx 5 \mathrm{nT}$ due to imperfectly compensated fringe fields were removed prior to graphing the results. The sample structure is well resolved for all selection fields used, and one clearly observes the increase of resolution with the magnitude of the gradient field. With the smallest of the used gradients, the system is close to the Sparrow's resolution limit where the three peaks are barely distinguishable without signal deconvolution. This means that with a gradient of $0.75 \mathrm{~T} / \mathrm{m}$ the spatial resolution of the raw image (width of the blurring kernel) $\delta x_{\text {raw }}$ is below $6 \mathrm{~mm}$. The spatial resolution increases linearly with the selection field strength, resulting in an estimated resolution of our MPIS system of

$$
\delta x_{\text {raw }}\left(G_{x}\right) \lesssim \frac{4.5 \mathrm{mT}}{G_{x}}
$$

for Resovist ${ }^{\circledR}$ nanoparticles. This results in estimated resolutions, prior to deconvolution, of $\delta x_{\text {raw }} \lesssim 4.5$ and $3.1 \mathrm{~mm}$ for $G_{x}=1$ and $1.4 \mathrm{~T} / \mathrm{m}$, respectively. As a comparison, the $\mathrm{x}$-space implementation of MPI with Resovist ${ }^{\circledR}$ and excitation field at $19 \mathrm{kHz}$ has a spatial resolution versus gradient $\delta x \approx 6.5 \mathrm{mT} / G_{x}$ [59]. This significant difference is due to the fact that the AM-detection at low excitation frequency is sensitive also to agglomerated particles [57]. The latter demonstrates the sensitivity of our system to a larger fraction of 
iron mass in Resovist ${ }^{\circledR}$ compared to standrad MPI techniques, as well as the possibility to use larger (or agglomerated) particles.

We remind that the spatial resolution is proportional to the characteristic field of the MNP which, in turn, is inversely proportional to the MNP's core volume. We also note note that the signal amplitude increases with decreasing gradient strength, since a smaller gradient increases the spatial extension, i.e., the number of magnetically unsaturated particles which contribute to the recorded susceptibility signal.

\section{Reconstruction of the SPION Distribution}

Because of the excellent quality of the raw experimental data we were able to reconstruct the source distribution by the following deconvolution algorithm. For this we have divided the FOV into $p$ identical $^{1}$ pixels of size $\delta x_{\text {pix }}$. The $i$-th pixel is denoted by the coordinate $x_{i}$ of its center, so that the FOV is described by the set of coordinates

$\mathrm{FOV}=\left(x_{1}, x_{2}, \ldots, x_{p-1}, x_{p}\right)$ with $\delta x_{\mathrm{stp}}=x_{i}-x_{i-1}$.

The SPION distribution is parametrized as $s_{\text {pix }}=\left\{n_{1}, \ldots, n_{p}\right\}$ where $n_{i}=N_{i} / \delta x_{\text {stp }}$ denotes the of MNP density in the ith pixel, $N_{i}$ being the total amount of SPIONs in that pixel. The expected signal $\delta B_{\chi_{\mathrm{AM}}}^{\text {theo }}\left(x_{s}\right)$ is then given by the discrete convolution

$$
\delta B_{\chi_{\mathrm{AM}}}^{\text {theo }}\left(x_{s}\right)=\delta x_{\text {stp }} \sum_{i=1}^{p} n_{i} \operatorname{PSF}_{\text {mech }}\left(x_{s}-x_{i}\right) .
$$

Fitting this model to the experimental data with the MNP densities $n_{i=1 \ldots p}$ as fit parameters, then yields the discrete spatial distribution $s_{\text {pix }}^{\text {deconv }}$.

For the deconvolution we have chosen a FOV discretization with $p=89$ that corresponds to $\sim 0.27 \mathrm{~mm}$-sized pixels. We have used Mathematica ${ }^{\circledR}$ (Wolfram, ver. 11.1) to fit the theoretical model (13) to the three experimental data sets of Fig. 6. The $\mathrm{PSF}_{\text {mech }}$ function was completely defined for each of the three fits based on the known experimental parameters. The deconvoluted distributions $s_{\text {pix }}^{\text {deconv }}$ are shown in Fig. $6 \mathrm{We}$ note that the three-peak structure of the sample is well resolved in all reconstructed images. However, the size distributions (blue histograms) derived from the data with the strongest applied gradient exhibit some deformation and broadening with respect to the anticipated result. This feature is due to the fact that in larger gradients the PSF becomes narrower, implying that less particles contribute to the instantaneous signal, which leads to a reduction of the $\mathrm{S} / \mathrm{N}$ ratio.

The accuracy of the source distributions reconstructed from the data taken with the 0.75 and $1.0 \mathrm{~T} / \mathrm{m}$ gradients is excellent, resulting in a spatial resolution of the deconvoluted distributions below $1 \mathrm{~mm}$. We therefore conclude that large gradients are advantageous for applications in which a precise (timeand computation-intensive) image reconstruction has to be avoided, such as, e.g., for realtime in vivo visualizations. In other cases, such as, e.g., visualizations of in vitro samples,

\footnotetext{
${ }^{1}$ In principle it is not necessary that the pixel sizes are all identical. With some a priori knowledge it may by advantageous to use another type of discretization.
}

TABLE I

AMOUNT OF MNP PER CAPILLARY (IN MILLIGRAMS OF IRON) OBTAINED BY INTEGRATING THE DATA SHOWN AS BLUE HISTOGRAMS IN FIG. 13

\begin{tabular}{l|ccc} 
& $x \approx-6 \mathrm{~mm}$ & $x \approx 0$ & $x \approx 6 \mathrm{~mm}$ \\
\hline$G_{x}=0.75 \mathrm{~T} / \mathrm{m}$ & 1.10 & 1.33 & 1.48 \\
$G_{x}=1.0 \mathrm{~T} / \mathrm{m}$ & 1.11 & 1.35 & 1.43 \\
$G_{x}=1.4 \mathrm{~T} / \mathrm{m}$ & 1.19 & 1.38 & 1.44
\end{tabular}

moderate gradients are superior, since they allow to reconstruct the source distribution with a higher fidelity.

Each peak in the images represents one of the three capillaries of the structured sample. The integral count of each histogram $\left(\sum_{i} n_{i}\right)$ is proportional to the SPION mass in the corresponding capillary. We have determined in this way the integral SPION amount in all peaks for all gradients. The results, given in Table I, show that the inferred SPION amounts in each capillary show a maximum spread of 8,3 , and $4 \%$ only for the different gradients used.

\section{ScAnNer PERformance}

\section{A. Magnetic Moment Detection Limit}

The magnetic moment detection limit (noise-equivalentmagnetic moment, NEM) $\delta \mu_{\mathrm{NEM}}$ of the AM-MPIS system is given by

$$
\delta \mu_{\mathrm{NEM}}=\frac{2 \pi}{\mu_{0}} z^{3} \delta B_{\mathrm{NEM}},
$$

where $\delta B_{\text {NEM }}$ is the AM's flux density detection limit (sensitivity), and $z$ the separation of the AM from the SPION sample. The proof-of-principle MPIS scanner presented here has a spectral noise density floor $\delta B_{\mathrm{NEM}}$ of $\approx 2 \mathrm{pT} / \sqrt{\mathrm{Hz}}$ at frequencies above $\sim 30 \mathrm{~Hz}$. Together with $z \sim 75 \mathrm{~mm}$, this yields a magnetic moment detection limit $\delta \mu_{\mathrm{NEM}}$ of $\approx 4.2 \mathrm{nA} \mathrm{m}^{2} / \sqrt{\mathrm{Hz}}$, that is comparable to the sensitivity of commercially available MPI scanners [60].

This estimation assumes an optimized MPIS scanner in which the magnetic noise (at the AM position) from the excitation coils is well below the AM detection limit. The magnetic moment detection limit defined in Eq. (14) depends then only on the AM performance and on the distance from the SPION sample.

Under reasonable assumptions concerning the further optimization of the described apparatus (e. g., by a better fringe field compensation and gradiometric detection with an anticipated sensitivity of $\approx 20 \mathrm{fT} / \sqrt{\mathrm{Hz}}$ ), we estimate that a magnetic moment detection limit $\delta \mu_{\mathrm{NEM}}$ of $\approx 40 \mathrm{pA} \mathrm{m}^{2} / \sqrt{\mathrm{Hz}}$ can be achieved, corresponding to a $500 \mathrm{nmol}_{\mathrm{Fe}} / 1$ SPION detection sensitivity for an averaging time of $30 \mathrm{~s}$.

\section{B. Spatial Resolution Limit}

The spatial resolution $\delta x_{\text {min }}$ of the raw image (i.e., prior to deconvolution) can be estimated as the minimal separation of two sources, above which a saddle point develops in the image (Sparrow criterion). For the Langevin model the resolution predicted by this criterion is $\delta x_{\min }=2.75 H_{k} / G_{x}=$ $3.55 \mathrm{mT} / G_{x}$, which is in good agreement with the empirical estimation (11). For the three experiments shown in Fig. 6 this expression results in resolutions $\delta x_{\min }$ of $4.75,3.55$, and 

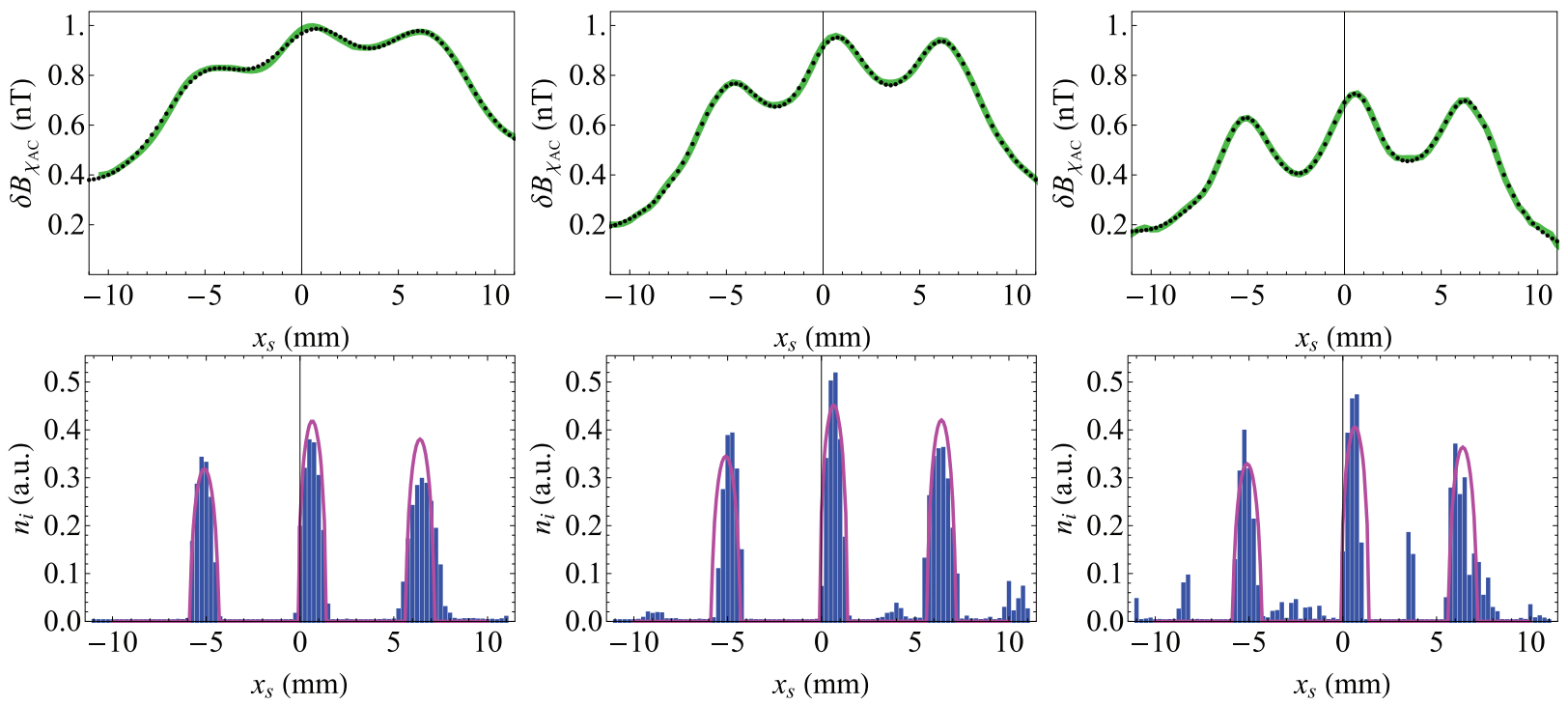

Fig. 6. Top: Raw 1D MPIS images of three nominally identical cylinder filled with Resovist ${ }^{\circledR}$, recorded in field gradients of 0.75 , 1.0 , and 1.4 T/m, respectively. Bottom: 1D density distribution inferred by deconvolution (blue histograms). The solid magenta lines represent the ideal $s(x)$ distributions (10) from cylindrical samples with diameter of $r_{i}=0.75 \mathrm{~mm}$. The magenta curves are normalized such that their integrals equal the integrals of the underlying histograms. In the top line the fit results (black dots) are superimposed on the measured signals (solid green lines).

$2.55 \mathrm{~mm}$, respectively. This limit can of course only be reached if a scan speed that is compatible with the system's detection bandwidth is used.

\section{Scan Speed and Bandwidth Limit}

The achievable detection bandwidth is determined by several factors. We recall that the AM proper (dashed box in Fig. 4) is operated as a phase-locked loop (PLL), whose typical bandwidth is $~ 5-10 \%$ of the local Larmor frequency (100 kHz in our case). In our device this bandwidth is $\approx 5 \mathrm{kHz}$, but we have artificially reduced it (using $\mathrm{LPF}_{1}$ in Fig. 4) to $1 \mathrm{kHz}$ in order to suppress the recorded noise power. The AM can thus follow magnetic field changes occurring at frequencies in the band of $0 \ldots 1 \mathrm{kHz}$.

In order to generate the derivative of the Langevin function we apply a modulation of the excitation field $H$ at a frequency $f_{\text {mod }}$ of $121 \mathrm{~Hz}$, which is well within the above bandwidth. The magnetometer signal (frequency proportional to $B_{\text {tot }}$ ) is demodulated with a second lock-in amplifier (right part of Fig. 4) using a bandwidth determined by the filter $\mathrm{LPF}_{2}: 5$ or $10 \mathrm{~Hz}$ in our case. We refer to this bandwidth as 'detection bandwidth' $f_{\mathrm{BW}}$.

We can relate the allowable scan speed $v_{s}$ to the detection bandwidth $f_{\mathrm{BW}}$ by the following Fourier space argument. We assume a point-like SPION sample $s(x)=\delta(x)$ located in the center of a one-dimensional MPIS. At time $t$ the nanoparticles feel an instantaneous offset field $H_{\mathrm{off}}(t)=G_{x} v_{s}(t) t=$ $G_{x} x(t)$, where $v_{s}(t)$ is the FFP's velocity, $G_{x}$ the magnetic field gradient and $x(t)$ the FFP position.

Under these conditions the time-dependence of the pointspread function (9) becomes

$$
\begin{aligned}
\operatorname{PSF}(t) & =\mathcal{A} H_{k}^{-1} L^{\prime}\left(\frac{G_{x} v_{s} t}{H_{k}}\right) \\
& =\mathcal{A}\left(\frac{H_{k}}{\left(G_{x} v_{s} t\right)^{2}}-\operatorname{csch}^{2} \frac{G_{x} v_{s} t}{H_{k}}\right)
\end{aligned}
$$

where $\mathcal{A}$ is a constant which depends on the experimental geometry. We note that this function, like its primitive, the Langevin function, is not defined at $t=0$. In order to have a physical meaning the function should be defined and continuous at every instant $t$. By imposing continuity at $t=0$ the resulting Fourier transform of the point-spread function is then given by

$$
\mathcal{P S F}(f)=\mathcal{A} \frac{2 f H_{k} \pi^{2}}{G_{x}^{2} v_{s}^{2}}\left(\operatorname{coth} \frac{\pi^{2} H_{k} f}{G_{x} v_{s}}-1\right),
$$

which defines the frequency spectrum of the MPIS signal, having a peak amplitude, $\mathcal{P} \mathcal{S} \mathcal{F}(f=0)=2 \mathcal{A} /\left(G_{x} v_{s}\right)$.

We define the resolution bandwidth $\mathrm{BW}_{\text {res }}$ as the width of an equivalent flat top spectrum with the same integral, viz., $\int_{0}^{\mathrm{BW}_{\text {res }}} 1 \mathrm{~d} f \equiv \int_{0}^{\infty}[\mathcal{P} \mathcal{S} \mathcal{F}(f) / \mathcal{P} \mathcal{S} \mathcal{F}(0)]^{2} \mathrm{~d} f$, yielding

$$
\mathrm{BW}_{\mathrm{res}}=\frac{\left(\pi^{2}-6 \mathcal{Z}(3)\right)}{6 \pi^{2}} \frac{G_{x} v_{s}}{H_{k}} \simeq 0.0448 \frac{G_{x} v_{s}}{H_{k}},
$$

where $\mathcal{Z}(x)=\sum_{k=1}^{\infty} k^{-x}$ is the Riemann Zeta-function. Equation (17) can be solved for the scan speed, yielding

$$
v_{s}=\frac{\mathrm{BW}_{\text {res }}}{0.0448} \frac{H_{k}}{G_{x}}=8.13 \delta x \mathrm{BW}_{\text {res. }} .
$$

For a given experimental detection bandwidth $f_{\mathrm{BW}}$, one thus has to ensure that the experimental scan speed obeys

$$
v_{s}^{\text {exp. }} \ll 8.13 \delta x f_{\text {BW }}
$$

in order to obtain a non-distorted image.

An experimental demodulation bandwidth that is too close to the estimated $\mathrm{BW}_{\text {res }}$ will cut high-frequency components from the PSF, leading to distortions. In principle one can take this into account by defining a modified PSF (multiplication of the theoretical PSF with the low-pass filter's transfer function), and using this modified PSF for the deconvolution. For ease of analysis, it is however advisable to use a bandwidth that is 
TABLE II

Scan Speeds Derived From Eq. (19), Compared to EXPERIMENTALLY USED SCAN SPEEDS DEPLOYED FOR RECORDING THE THREE SAMPLE ScANS OF FIG. 6. The Rightmost Columns Gives the MAXIMAL Achievable Frame Rates According to EQ. (20)

\begin{tabular}{ccccc}
$\begin{array}{c}G_{x} \\
\mathrm{~T} / \mathrm{m}\end{array}$ & $\begin{array}{c}f_{\mathrm{BW}} \\
\mathrm{Hz}\end{array}$ & $\begin{array}{c}v_{s} \text { from Eq. (19) } \\
\mathrm{mm} / \mathrm{s}\end{array}$ & $\begin{array}{c}v_{s}^{\text {exp. }} \\
\mathrm{mm} / \mathrm{s}\end{array}$ & $\begin{array}{c}R_{\text {frame }}^{\max } \\
\mathrm{s}^{-1}\end{array}$ \\
\hline 0.75 & 5 & 193 & 7.5 & 24 \\
1 & 5 & 144 & 7.5 & 18 \\
1.4 & 8 & 163 & 7.5 & 13
\end{tabular}

much larger than $\mathrm{BW}_{\text {res }}$, which we did, as evidenced by the values given in Table II.

The minimal demodulation bandwidth further defines the minimal modulation frequency, since lock-in demodulation requires that $f_{\text {mod }} \gg \mathrm{BW}_{\text {res }}$ must be obeyed.

The achievable frame rate can be estimated from (19) as

$$
R_{\text {frame }}=\frac{v_{s}^{\text {exp. }}}{\text { FOV }}<R_{\text {frame }}^{\max } \approx \frac{\delta x}{\text { FOV }} f_{\text {mod }},
$$

assuming that one can achieve an experimental bandwidth $f_{\mathrm{BW}}$ of $10 \%$ of $f_{\text {mod }}$. Numerical values for the maximal frame rate $R_{\text {frame }}^{\max }$ are given in Table II.

\section{Further Development and Upscaling of MPIS}

A fundamental limitation to the upscaling of the AM-based instrument lays in the fast $\left(z_{\mathrm{AM}}^{-3}\right)$ decay of the detected flux density produced by the small dipolar source with its distance, $z_{\mathrm{AM}}$, to the sensor since the AM has to be located outside of the FOV. This problem can be partially resolved by placing several AM sensors around the object at the boundaries of the FOV, and whenever possible designing the instrument with a "slab-like" FOV, in order to minimize the distance to the induction source. Suitable AM arrays, spanning the human chest area, have been successfully demonstrated in cardiomagnetometry applications [5], [6]. An AM-array-based scanner will further deliver a stable, FOV-independent scanning speed since each AM will track signals only from a partial FOV in its close vicinity, while all sensors will be recording data in parallel.

In principle, MPIS should be compatible with various single-sided MPI systems [61]-[63], which are designed to work in shallow depths and a relatively small FOV. It might thus be envisioned to integrate MPIS with an animalsize or even human-size MPI. One may, furthermore, avoid an upscaling of the encoding gradient coil system, by replacing a very large coil by an array of the scanner coils, similar to the ones used in single-sided MPI scanner [64]. A major obstacle for implementing this approach lies in the design of appropriately self-shielded coils that do not degrade the AM performance. Enhancing the robustness of the AM sensor proper against fringe fields and field gradients may be a more efficient approach for circumventing this obstacle. In fact (cf. Sec. IV), the tolerance of the AM sensor to the range and speed of change of the fringing magnetic field is currently constrained by the bandwidth of the PLL-loop controlling the spin oscillation in the sensor cell. We have performed initial tests of a similar AM sensor in a much faster feedback mode, which promises at least one order of magnitude larger Larmor precession tracking bandwidth and a corresponding increase in fringe field tolerance.

Although further investigations are necessary to evaluate the performance of such improved sensors in an MPIS setup, we believe that the practical realization of MPIS scanners for object of tens of cm may come within reach.

\section{CONCLUSION}

We have presented a proof-of-principle mechanical 1D MPI scanner, based on a single atomic magnetometer (AM) as signal detector. In view of the big variety of anticipated AM-based MPI implementations, we have realized a scanning AC-susceptometer, with which magnetic signals can be analyzed based on the (separately measured) $M(H)$ response curve of the deployed SPIONs in order to infer SPION density distributions. In a series of experimental studies we have quantified the performance of the AM-MPIS instrument and have shown that microscopic amounts of SPIONs can be localized with sub-mm accuracy in a direct, computationally undemanding, and potentially on-line manner. We have further investigated how tuning the used field gradient affects the instruments resolution and sensitivity. Our MPI design modality allows the use of larger-sized (or agglomerated) SPIONs than those deployed in conventional MPI scanners, thereby allowing a considerable reduction of the required gradient strength for a given spatial resolution, entities to which classical receiver-coil based MPI scanners are mostly blind. Our scanner implementation is further not limited to AM sensing, but can - in principle - operate with any high sensitivity magnetometer whose bandwidth reaches down to (near) DC, such as SQUIDs, fluxgates or GMR (giant magneto-resistivity) devices. In this way, MPIS may open new ways towards the development of clinical scanners that circumvent the SAR-related problems of existing devices.

\section{ACKNOWLEDGMENT}

The authors would like to thank Dr. Vladimir Dolgovskiy for useful discussions on source reconstruction techniques, Dr. Zoran D. Grujić and Dr. Theo Scholtes for technical assistance, and Dr. Uwe Steinhoff for valuable notes on SPION kinetics.

\section{References}

[1] D. Budker and D. F. J. Kimball, Optical Magnetometry. New York, NY, USA: Cambridge Univ. Press, 2013.

[2] S. Afach et al., "Highly stable atomic vector magnetometer based on free spin precession," Opt. Exp., vol. 23, no. 17, pp. 22108-22115, Aug. 2015. [Online]. Available: http://www.opticsexpress.org/abstract. cfm?URI=oe-23-17-22108

[3] S. Pustelny et al., "The global network of optical magnetometers for exotic physics (GNOME): A novel scheme to search for physics beyond the standard model," Annalen der Physik, vol. 525, nos. 8-9, pp. 659-670, 2013.

[4] I. Fratter et al., "Swarm absolute scalar magnetometers first in-orbit results," Acta Astronautica, vol. 121, pp. 76-87, Apr./May 2016. [Online]. Available: http://www.sciencedirect.com/science/article/pii/ S0094576515004671 
[5] G. Bison et al., "A room temperature 19-channel magnetic field mapping device for cardiac signals," Appl. Phys. Lett., vol. 95, no. 17, Oct. 2009 , Art. no. 173701. doi: 10.1063/1.3255041.

[6] G. Lembke, S. N. Erné, H. Nowak, B. Menhorn, A. Pasquarelli, and G. Bison, "Optical multichannel room temperature magnetic field imaging system for clinical application," Biomed. Opt. Express vol. 5, no. 3, pp. 876-881, Mar. 2014. [Online]. Available: http:// www.osapublishing.org/boe/abstract.cfm?URI=boe-5-3-876

[7] O. Alem et al., "Fetal magnetocardiography measurements with an array of microfabricated optically pumped magnetometers," Phys. Med. Biol., vol. 60, no. 12, pp. 4797-4811, 2015.

[8] T. H. Sander, J. Preusser, R. R. Mhaskar, J. E. Kitching, L. Trahms, and S. Knappe, "Magnetoencephalography with a chip-scale atomic magnetometer," Biomed. Opt. Express, vol. 3, no. 5, pp. 981-990, 2012.

[9] S. Knappe, T. Sander, and L. Trahms, Optically-Pumped Magnetometers for MEG. Berlin, Germany: Springer, 2014, pp. 993-999. doi: 10.1007/978-3-642-33045-2 49.

[10] K. Kamada et al., "Human magnetoencephalogram measurements using newly developed compact module of high-sensitivity atomic magnetometer," Jpn. J. Appl. Phys., vol. 54, no. 2, 2015, Art. no. 026601. [Online]. Available: http://stacks.iop.org/ $1347-4065 / 54 / \mathrm{i}=2 / \mathrm{a}=026601$

[11] E. Boto et al., "Moving magnetoencephalography towards real-world applications with a wearable system," Nature, vol. 555, pp. 657-661, Mar. 2018. [Online]. Available: http://dx.doi.org/10.1038/nature26147

[12] A. Jodko-Władzińska et al., "Individualized magnetoencephalography using optically pumped magnetometers with an anatomy derived sensor holder," Biomed. Eng. Biomed. Tech., vol. 63, no. S1, p. 240, 2018 doi: $10.1515 / \mathrm{bmt}-2018-6045$.

[13] K. Jensen et al., "Non-invasive detection of animal nerve impulses with an atomic magnetometer operating near quantum limited sensitivity," Sci. Rep., vol. 6, Jul. 2016, Art. no. 29638. doi: 10.1038/srep29638.

[14] I. Savukov and T. Karaulanov, "Magnetic-resonance imaging of the human brain with an atomic magnetometer," Appl. Phys. Lett., vol. 103 , no. 4, 2013, Art. no. 043703. doi: 10.1063/1.4816433.

[15] C. Johnson et al., "Magnetic relaxometry with an atomic magnetometer and squid sensors on targeted cancer cells," J. Magn. Magn. Mater. vol. 324, no. 17, pp. 2613-2619, Aug. 2012. [Online]. Available: http://www.ncbi.nlm.nih.gov/pmc/articles/PMC3389787/

[16] V. Dolgovskiy et al., "A magnetic source imaging camera," Appl. Phys. Lett., vol. 109, no. 2, 2016, Art. no. 023505. doi: 10.1063/1.4958700.

[17] T. Oida, K. Kato, Y. Ito, and T. Kobayashi, "Remote detection of magnetic signals with a compact atomic magnetometer module towards human MRI-MPI hybrid systems," Int. J. Magn. Part. Imag., vol. 4, no. 2, 2019, Art. no. 1906001. [Online]. Available: https://journal.iwmpi.org/index.php/iwmpi/article/view/130

[18] I. K. Kominis, T. W. Kornack, J. C. Allred, and M. V. Romalls, "A subfemtotesla multichannel atomic magnetometer," Nature, vol. 422, p. 596, Apr. 2003. doi: 10.1038/nature01484

[19] S. Knappe, T. H. Sander, O. Kosch, F. Wiekhorst, J. Kitching, and L. Trahms, "Cross-validation of microfabricated atomic magnetometers with superconducting quantum interference devices for biomagnetic applications," Appl. Phys. Lett., vol. 97, no. 13, 2010, Art. no. 133703. doi: 10.1063/1.3491548.

[20] I. M. Savukov, S. J. Seltzer, M. V. Romalis, and K. L. Sauer, "Tunable atomic magnetometer for detection of radio-frequency magnetic fields," Phys. Rev. Lett., vol. 95, no. 6, 2005, Art. no. 063004.

[21] S.-K. Lee, K. L. Sauer, S. J. Seltzer, O. Alem, and M. V. Romalis, "Subfemtotesla radio-frequency atomic magnetometer for detection of nuclear quadrupole resonance," Appl. Phys. Lett., vol. 89, no. 21, 2006, Art. no. 214106. doi: 10.1063/1.2390643.

[22] W. Chalupczak, R. M. Godun, and S. Pustelny, "Radiofrequency spectroscopy as a tool for studying coherent spin dynamics and for application to radio-frequency magnetometry,' Adv. At., Mol., Opt. Phys., vol. 67, pp. 297-336, Jan. 2018 [Online]. Available: http://www.sciencedirect.com/science/article/ pii/S1049250X18300053

[23] A. Horsley and P. Treutlein, "Frequency-tunable microwave field detection in an atomic vapor cell," Appl. Phys. Lett., vol. 108, no. 21, 2016 Art. no. 211102. doi: 10.1063/1.4950805.

[24] J. M. Higbie, E. Corsini, and D. Budker, "Robust, high-speed, alloptical atomic magnetometer," Rev. Sci. Instrum., vol. 77, no. 11, 2006 , Art. no. 113106. doi: 10.1063/1.2370597.
[25] S. Gross, C. Barmet, B. E. Dietrich, D. O. Brunner, T. Schmid, and K. P. Pruessmann, "Dynamic nuclear magnetic resonance field sensing with part-per-trillion resolution," Nature Commun., vol. 7, Dec. 2016, Art. no. 13702.

[26] B. Gleich and J. Weizenecker, "Tomographic imaging using the nonlinear response of magnetic particles," Nature, vol. 435, pp. 1214-1217, Jun. 2005.

[27] S. Colombo, V. N. Lebedev, A. Tonyushkin, Z. D. Grujic, V. Dolgovskiy, and A. Weis, "Towards a mechanical MPI scanner based on atomic magnetometry," Int. J. Magn. Part. Imag., vol. 3, no. 1, 2017, Art. no. 1703006.

[28] J. Weizenecker, B. Gleich, and J. Borgert, "Magnetic particle imaging using a field free line," J. Phys. D, Appl. Phys., vol. 41, May 2008, Art. no. 105009.

[29] Q. A. Pankhurst, J. Connolly, S. K. Jones, and J. Dobson, "Applications of magnetic nanoparticles in biomedicine," J. Phys. D, Appl. Phys., vol. 36, no. 13, p. R167, 2003.

[30] A.-H. Lu, E. E. Salabas, and F. Schüth, "Magnetic nanoparticles: Synthesis, protection, functionalization, and application," Angew. Chem. Int. Ed., vol. 46, no. 8, pp. 1222-1244, 2007.

[31] J. Franke et al., "System characterization of a highly integrated preclinical hybrid MPI-MRI scanner," IEEE Trans. Med. Imag., vol. 35, no. 9 , pp. 1993-2004, Sep. 2016

[32] P. Vogel et al., "MRI meets MPI: A bimodal MPI-MRI tomograph," IEEE Trans. Med. Imag., vol. 33, no. 10, pp. 1954-1959, Oct. 2014.

[33] E. U. Saritas et al., "Magnetic particle imaging (MPI) for NMR and MRI researchers," J. Magn. Reson., vol. 229, pp. 116-126, Apr. 2013. [Online]. Available: http://www.sciencedirect.com/science/ article/pii/S1090780712003734

[34] M. S. Judenhofer et al., "Simultaneous PET-MRI: A new approach for functional and morphological imaging," Nature Med., vol. 14, pp. 459-465, Mar. 2008. doi: 10.1038/nm1700.

[35] Bruker. (2014). The World'S First Preclinical MPI System [Online]. Available: https://www.bruker.com/fileadmin/user_upload/8 PDF-Docs/PreclinicalImaging/Brochures/MPI-PreClinical-Brochure.pdf

[36] (2017). Momentum TM Imager Magnetic Particle Imaging System. [Online]. Available: https://www.magneticinsight.com/momentumimager/

[37] E. U. Saritas, P. W. Goodwill, and S. M. Conolly, "Effects of pulse duration on magnetostimulation thresholds," Med. Phys., vol. 42, no. 6, pp. 3005-3012, 2015.

[38] O. B. Demirel and E. U. Saritas, "Effects of duty cycle on magnetostimulation thresholds in MPI," Int. J. Magn. Part. Imag., vol. 3, no. 1, 2017, Art. no. 703010. [Online]. Available: https://journal.iwmpi.org/index.php/iwmpi/article/view/48

[39] O. Dössel and J. Bohnert, "Safety considerations for magnetic fields of $10 \mathrm{mt}$ to $100 \mathrm{mt}$ amplitude in the frequency range of $10 \mathrm{khz}$ to $100 \mathrm{khz}$ for magnetic particle imaging," Biomed. Eng., vol. 58, no. 6, pp. 611-621, 2013.

[40] P. W. Goodwill et al., "X-space MPI: Magnetic nanoparticles for safe medical imaging," Adv. Mater., vol. 24, pp. 3870-3877, Jul. 2012.

[41] E. U. Saritas, P. W. Goodwill, G. Z. Zhang, and S. M. Conolly, "Magnetostimulation limits in magnetic particle imaging," IEEE Trans. Med. Imag., vol. 32, no. 9, pp. 1600-1610, Sep. 2013.

[42] H. Arami et al., "Tomographic magnetic particle imaging of cancer targeted nanoparticles," Nanoscale, vol. 9, no. 47, pp. 18723-18730, Oct. 2017.

[43] K. Enpuku et al., "Wash-free detection of C-reactive protein based on third-harmonic signal measurement of magnetic markers," Jpn. J. Appl. Phys., vol. 57, Aug. 2018, Art. no. 090309. [Online]. Available: http://stacks.iop.org/1347-4065/57/i=9/a=090309

[44] W. F. Brown, Jr., "Thermal fluctuations of a single-domain particle," Phys. Rev., vol. 130, p. 1677, Jun. 1963.

[45] M. A. Martsenyuk, Y. L. Raikher, and M. I. Shliomis, "On the kinetics of magnetization of suspensions of ferromagnetic particle," J. Exp. Theor. Phys., vol. 38, no. 2, pp. 413-416, 1973.

[46] J. Rahmer, J. Weizenecke, B. Gleich, and J. Borgert, "Signal encoding in magnetic particle imaging: Properties of the system function," $B M C$ Med. Imag., vol. 9, Dec. 2009, Art. no. 4.

[47] J. Rahmer, J. Weizenecke, B. Gleich, and J. Borgert, "Analysis of a 3-D system function measured for magnetic particle imaging," IEEE Trans. Med. Imag., vol. 31, no. 6, pp. 1289-1299, Jan. 2012. 
[48] Z. W. Tay, D. W. Hensley, E. C. Vreeland, B. Zheng, and S. M. Conolly, "The relaxation wall: Experimental limits to improving MPI spatial resolution by increasing nanoparticle core size," Biomed. Phys. Eng. Express, vol. 3, no. 3, 2017, Art. no. 035003.

[49] Z. W. Tay et al., "Pulsed excitation in magnetic particle imaging," IEEE Trans. Med. Imag., to be published.

[50] H. Arami, R. M. Ferguson, A. P. Khandhar, and K. M. Krishnan, "Sizedependent ferrohydrodynamic relaxometry of magnetic particle imaging tracers in different environments," Med. Phys., vol. 40, no. 7, 2013, Art. no. 071904

[51] C. Kuhlmann et al., "Drive-field frequency dependent MPI performance of single-core magnetite nanoparticle tracers," IEEE Trans. Magn., vol. 51, no. 2, pp. 1-4, Feb. 2015

[52] A. Tonyushkin and M. Prentiss, "Straight macroscopic magnetic guide for cold atom interferometer," J. Appl. Phys., vol. 108, Nov. 2010, Art. no. 094904

[53] A. Tonyushkin, "Novel selection coils design for 3D FFL-based MPI," in Proc. 6th Int. Workshop Magn. Part. Imag., T. M. Buzug, J. Borgert, and T. Knopp, Eds. Hingham, MA, USA: Infinite Science, Mar. 2016 , p. 33 .

[54] A. Kastler, "Optical methods of atomic orientation and of magnetic resonance," J. Opt. Soc. Amer., vol. 47, no. 6, pp. 460-465, 1957.

[55] O. Chubar, P. Elleaume, and J. Chavanne, "A three-dimensional magnetostatics computer code for insertion devices," J. Synchrotron Radiat., vol. 5, no. 3, pp. 481-484, 1998.

[56] D. Eberbeck, C. L. Dennis, N. F. Huls, K. L. Krycka, C. Grüttner, and F. Westphal, "Multicore magnetic nanoparticles for magnetic particle imaging," IEEE Trans. Magn., vol. 49, no. 1, pp. 269-274, Jan. 2013.
[57] S. Colombo, V. Lebedev, Z. D. Grujic, V. Dolgovskiy, and A. Weis, "M(H) dependence and size distribution of SPIONs measured by atomic magnetometry," Int. J. Magn. Part. Imag., vol. 2, no. 1, 2016, Art. no. 1606002. [Online]. Available: https://journal. iwmpi.org/index.php/iwmpi/article/view/28

[58] S. Colombo, V. Lebedev, Z. D. Grujic, V. Dolgovskiy, and A. Weis, "MPS and ACS with an atomic magnetometer," Int. J. Magn. Part. Imag., vol. 2, no. 1, 2016, Art. no. 1604001.

[59] P. W. Goodwill, K. Lu, B. Zheng, and S. M. Conolly, "An X-space magnetic particle imaging scanner," Rev. Sci. Instrum., vol. 83, no. 3 , 2012, Art. no. 033708. doi: 10.1063/1.3694534.

[60] H. Paysen et al., "Towards quantitative magnetic particle imaging: A comparison with magnetic particle spectroscopy," AIP $A d v$., vol. 8 , no. 5, 2018, Art. no. 056712. doi: 10.1063/1.5006391.

[61] C. Kaethner, M. Ahlborg, K. Gräfe, G. Bringout, T. F. Sattel, and T. M. Buzug, "Asymmetric scanner design for interventional scenarios in magnetic particle imaging," IEEE Trans. Magn., vol. 51, no. 2, Feb. 2015, Art. no. 6501904

[62] A. Tonyushkin, "Single-sided field-free line generator magnet for multidimensional magnetic particle imaging," IEEE Trans. Magn., vol. 53, no. 9, Sep. 2017, Art. no. 5300506.

[63] C. Z. Cooley, J. B. Mandeville, E. E. Mason, E. T. Mandeville, and L. L. Wald, "Rodent cerebral blood volume (CBV) changes during hypercapnia observed using magnetic particle imaging (MPI) detection,' NeuroImage, vol. 178, pp. 713-720, Sep. 2018.

[64] K. Gräfe, A. von Gladiss, G. Bringout, M. Ahlborg, and T. M. Buzug, "2D images recorded with a single-sided magnetic particle imaging scanner," IEEE Trans. Med. Imag., vol. 35, no. 4, pp. 1056-1065, Apr. 2016. 